\title{
Atrial voltage mapping: tailoring ablation approach by targeting the arrhythmogenic substrate
}

\author{
Vassil Traykov \\ Acibadem City Clinic Tokuda Hospital, Sofia, Bulgaria \\ Address for correspondence: \\ Department of Invasive Electrophysiology, Acibadem City Clinic Tokuda Hospital \\ N. Vaptzarov blvd. 51B, 1407 Sofia, Bulgaria. \\ E-mail: vtraykov@yahoo.com
}

The interplay between triggers, arrhythmogenic substrate and modulators determines the pathophysiological and clinical course of atrial fibrillation. Catheter ablation is currently the mainstay of therapy of this complex arrhythmia. Although mainly based on pulmonary vein isolation empirical ablation approaches targeting extrapulmonary substrate have also been introduced. These include creation of linear lesions, extensive ablation of LA posterior wall, targeting fractionated electrograms, rotors maintaining AF, etc. However, many of these approaches are just empirical and do not provide an individualised approach to each patient. Recently, the concept of fibrotic atrial cardiomyopathy has been introduced to explain the occurrence and clinical course of many conditions including AF. Atrial voltage mapping is increasingly recognised as a means to identify those fibrotic areas. Targeting low voltage zones in the atria during catheter ablation of AF seems to be associated with better clinical outcomes. This review focuses on the association between low-voltage zones and atrial fibrosis. It covers many of the technical details and limitations of voltage mapping to identify atrial substrate. It also summarises the available data from clinical trials examining the role of voltage-driven ablation on procedural outcome on AF patients.

Keywords: atrial fibrillation, voltage mapping, ablation, atrial fibrosis

\section{Introduction}

From the pathophysiological standpoint atrial fibrillation (AF) is a complex disease bringing together three major mechanisms for its initiation and maintenance. These are the triggers, the arrhythmogenic substrate and multiple modulators. When trigger activity is the single or predominant mechanism of AF occurrence in the absence of a significant arrhythmogenic substrate then the AF might be considered a self-perpetuating process, as stated by the "AF begets AF" concept by Wijffels et al. (1). However, in most of the cases there is interplay between triggers and arrhythmogenic substrate. In these cases, pre-existing fibrotic changes in the atria might be responsible for creating conditions for the triggering activity to initiate AF episodes. Such a scenario might even question the existence of lone AF as defined by lack of structural heart disease in young patients (2).

Currently catheter ablation is the mainstay treatment modality in atrial fibrillation (AF) $(3,4)$. Since its introduction at the end of the 90-s by Haissaguerre et al. pulmonary vein isolation $(\mathrm{PVI})$ has turned into the cornerstone of this treatment modality (5). It is the main and frequently the only step undertaken during ablation of paroxysmal and persistent AF. Results from PVI as a stand-alone procedure in patients with paroxysmal AF are quite promising in achieving long-term freedom 
from arrhythmias in up to $94 \%$ especially after the introduction of recent technological advancements and new ablation algorithms (6). Long-term success rates of PVI in non-paroxysmal AF (NPAF) are lower according to large randomised trials (7). In NPAF additional ablation approaches have been proposed. These include stepwise ablation, defragmentation by targeting complex fractionated electrograms, linear ablation, rotor modulation and even combined catheter ablation with alcohol ablation (8-11). All these techniques have been proven to be effective in small or single centre studies but never showed superiority in large randomised controlled trials (7). One potential explanation for this could be that most of them are empirically applied to individual patients all of whom might have different substrates. Recently the term atrial cardiomyopathy was introduced to describe a specific entity defined as: 'any complex of structural, architectural, contractile or electrophysiological changes affecting the atria with the potential to produce clinically-relevant manifestations' (12). These are a result of many pathological conditions but share many morphological similarities. The presence of such fibrotic atrial cardiomyopathy could explain a myriad of different clinical conditions - from AF and atrial tachycardia to sinus node disease and even thromboembolic complications (13).

Identifying and targeting fibrotic areas during ablation seems to be a promising approach to tailoring ablation therapy to fit the specific substrate in each individual patient. The current review will discuss some issues on the association of atrial fibrosis with clinical type of AF, then summarise the available evidence on the association of left atrial fibrosis on the outcome of AF ablation. Further on it will focus on the methods to identify fibrotic areas based on atrial voltage mapping covering most of its technical aspects. Finally, the review will summarise the existing data from clinical trials examining the role of voltage-guided ablation on procedural outcome on AF patients.

\section{AF phenotype and potential pitfalls of non-tailored approach to AF ablation}

The landmark concept of "AF begets AF" postulates that AF episodes would change electrical properties of atrial myocardium thus facilitating further AF occurrence (1). In an animal model the authors showed that bursts of high rate atrial pacing are able induce and maintain AF episodes of progressively longer duration. This was accompanied by marked changes in electrophysiological properties of the atrial myocardium. Such findings might serve to explain the generally accepted principle that AF occurs initially as a paroxysmal arrhythmia and over time it progresses into persistent AF of different duration (3). Many studies in humans have also supported these findings. However, this "natural evolution" of AF is not always so straightforward and there are series describing patients with AF initially presenting as persistent cases and never going through the "paroxysmal phase". On the other hand, many paroxysmal AF cases never progress to persistent AF despite the presence of very frequent episodes. Actually several groups have investigated the characteristics of patients with persistent AF "early in the course" or "directly from the onset" demonstrating characteristics suggesting more expressed arrhythmogenic substrate and poor outcome of the ablation $(14,15)$. There is also data showing very advanced atrial fibrosis in patients with paroxysmal AF and only mild fibrotic changes in persistent AF patients as assessed by histological studies and markers of collagen synthesis (16). Mahnkopf et al. demonstrated that the extent of LA fibrosis as assessed by late enhancement on magnetic resonance imaging (DE-MRI) is not dependent on AF type and the presence of comorbidities (2). In another series Stiles et al. report structural and electrophysiological abnormalities even in patients with "lone" paroxysmal AF (17). These findings question the concept that AF burden or persistence play a significant role in the development of atrial fibrosis. Therefore, from the standpoint of the degree of arrhythmogenic substrate the mechanistic division of AF into paroxysmal and persistent forms seems to be inappropriate.

Selecting patients who would require substrate modification as an additive to PVI based only on clinical presentation might not be entirely justified. Applying more extensive empirical ablation without identifying the underlying arrhythmogenic substrate may lead to overtreatment with subsequent increase in procedure duration, X-ray exposure and increased complication rate. Furthermore, some patients would remain undertreated and show more arrhythmia recurrences.

\section{Atrial voltage as a marker of atrial fibrosis}

The term arrhythmogenic substrate has been used to represent all the structural changes in the atrial myocardium which predispose to AF - LA fibrosis, atrial dysfunction, ultrastructural changes, etc. Obviously, imaging would represent the modality of choice to identify atrial fibrosis. Of the available imaging modalities magnetic resonance imaging (MRI) has been put forward as a means to assess LA fibrosis. Several studies demonstrate the value of delayed enhancement MRI (DE-MRI) to delineate the areas of LA fibrosis. However, this imaging modality is not completely standardized, and it comes with many pitfalls and unresolved issues. It usually requires considerable postprocessing using dedicated software (2). Atrial fibrosis assessed by histological examination has been found to correlate with zones of DE-MRI as shown by McGann et al. (18). Numerous studies using DE-MRI have demonstrated different 
outcomes of AF ablation based on extent of delayed enhancement on $\operatorname{MRI}(2,18,19)$. Based on the extent of LA fibrosis expressed as a percentage of zones of DE-MRI compared to the area of the whole LA the Utah classification (Utah I to IV) have been introduced.

Whether atrial bipolar voltage correlates with the areas of late enhancement has been studied by many groups. Spragg et al. have demonstrated that areas of DE-MRI showed lower voltage as compared to areas with normal MRI characteristics (20). They studied 10 patients undergoing redo AF ablation. Electroanatomical LA maps were created for each patient and co-registered with a preacquired MRI scan. The mean voltage of points identified as scar by MRI was lower than the voltage of points taken from non-scar regions $(0.39 \pm 0.61 \mathrm{mV}$ vs. $1.38 \pm 1.23 \mathrm{mV}, P<0.01)$. DE-MRI in this study was found to identify areas of low voltage $(0.5 \mathrm{mV})$ with a sensitivity of $84 \%$ and specificity of $68 \%$. Another study investigates the atrial voltage in 21 patients with paroxysmal AF by creating a high-density electro-anatomical map of the LA (21). The acquired voltage maps were registered together with the preacquired MRI models. Their results show that increasing levels of late gadolinium enhancement were associated with progressively lower endocardial voltage. Not all studies, however, report such a straightforward relationship. For instance, a study by Jadidi et al. examined 18 patients with persistent AF in an attempt to correlate the areas of LA fibrosis as assessed by MRI to zones of fractionated EGMs. They found that the mean voltage in the zones of dense late gadolinium enhancement was only slightly, but significantly lower than the ones recorded in the non-fibrotic regions $(0.6 \pm 0.8 \mathrm{mV}$ vs. $0.86 \pm 0.89 \mathrm{mV}$, $\mathrm{P}<0.001)$. Khurram et al. have studied 75 patients with $\mathrm{AF}$ in order to clarify the correlation between abnormal voltage and the presence and distribution of LA fibrosis (22). They have designed a specific MRI parameter the regional image intensity ratio (IIR) which represents the ratio between the mean pixel intensity for each sector (overall the LA area was divided into 20 sectors) divided by the mean pixel intensity of the whole LA blood pool. All the patients underwent detailed high-density endocardial LA voltage map during sinus rhythm before ablation. The results from this study show that IIR correlates strongly with bipolar LA voltage so that local IIR thresholds of $>0.97$ and $>1.61$ corresponded to atrial bipolar voltage $<0.5$ and $<0.1 \mathrm{mV}$, respectively. Following adjustment for some clinical characteristics and other covariates the authors report that each unit increase in local IIR was associated with a significant $91.3 \%$ reduction in local bipolar atrial voltage.

Several other factors have been shown to have an impact on the atrial voltage. Among these is LA size which was found to be associated with lower mean atrial voltage (23). A possible explanation for this is the potential link between atrial wall stress and morphological changes. A similar correlation has also been found between atrial pressure and mean atrial voltage (24). Many echocardiographic parameters of atrial function such as LA emptying fraction, total LA strain have also been shown to correlate with atrial voltage demonstrating the potential of these parameters to serve as indirect evidence for the presence of atrial fibrosis $(25,26)$.

\section{Defining atrial substrate by electro-anatomical voltage mapping}

Defining a universal threshold for the presence of atrial fibrosis in AF patients would represent a major breakthrough to the understanding and better identification of the arrhythmogenic substrate. Many centres accept the value of $0.5 \mathrm{mV}$ as an atrial voltage threshold to identify abnormal areas affected by atrial fibrosis although this finding lacks proper pathological validation. Available data is quite heterogeneous and demonstrates lack of a widely accepted voltage threshold that could serve to demonstrate abnormality. Most of the studies use the $5^{\text {th }}$ centile of the voltage at all the mapping points to define abnormalities.

Some studies have examined patients with supraventricular tachycardia undergoing LA mapping in an attempt to identify "normal" LA voltage in "healthy" patients and compare them to AF patients. The study by Yagishita et al. studied 6 patients with apparently "healthy" atria and demonstrated that $95 \%$ of the bipolar electrograms had bipolar voltage of $>1.17 \mathrm{mV}(27)$. In an elegant study Saghy et al. examined the association of fractionated electrograms in AF and in sinus rhythm in patients with persistent AF (28). This study included a control group of 9 patients with structurally normal hearts and non-AF substrates (WPW or supraventricular tachycardia). The authors found that in the control group $95 \%$ of all bipolar electrograms were $>0.5 \mathrm{mV}$. On the other hand, Teh et al. demonstrate in a group of 15 patients with supraventricular tachycardia that mean LA voltage decreases progressively among patients without structural heart disease and with supraventricular tachycardia $(2.8 \mathrm{mV})$, paroxysmal AF $(2.2 \mathrm{mV})$ and persistent AF (1.8 mV) (29). All these findings support the notion of a lack of a clear cut-off value for LA voltage to identify atrial substrate. To some extent the discrepant results, both in non-AF and in AF patients might be explained by different approach for mapping. Other studies attempt to determine the cut-off values for "normal" AF voltage in AF patients and use this value to analyse outcomes and other procedural and clinical endpoints. In a large series of 700 patients undergoing pulmonary vein isolation Verma et al. performed LA electroanatomical map searching for areas of LA scarring (30). In this study scar was defined as signal amplitude $<0.05 \mathrm{mV}$ and amplitudes $<0.5 \mathrm{mV}$ were used to define "abnormal" areas. Based on these definitions presence of LA scarring was found to be 
an independent predictor of arrhythmia recurrence following catheter ablation. In another series Kapa et al. studied 20 patients with AF (31). According to their results a voltage cut-off of $<0.2 \mathrm{mV}$ at the junction of LA and $\mathrm{PVs}$ and $<0.45 \mathrm{mV}$ at all other areas was useful to identify scar at these locations. In addition, a cut-off value of $0.27 \mathrm{mV}$ had the highest diagnostic accuracy to delineate scar as identified by MRI. A study by Rolf et al. also reports values higher than $0.5 \mathrm{mv}$ to represent "healthy" areas while values in the range 0.2 to 0.5 $\mathrm{mV}$ were considered diseased areas and voltage $<0.2$ $\mathrm{mV}$ was thought to represent atrial scar (32). Contrary to that, the work of Kottkamp et al. suggests different range of values with voltages $<0.5 \mathrm{mV}$ thought to represent areas of severe fibrosis, those between 0.5 and $1.5 \mathrm{mV}$ - zones of mild/moderate fibrosis and $>1.5 \mathrm{mV}$ would signify "less affected/normal" myocardium. In addition, scar was defined only at sites where there was evident lack of discreet electrical activity and inability to achieve local capture during pacing. As shown above this group introduced the term fibrotic atrial cardiomyopathy and established a classification based on the extent of abnormal areas: FACM 0 to 4 similar to the Utah classification (13, 33). An explanation for the apparently "liberal" definition of low voltage zones comes from the fact that this group has identified abnormal fragmented electrograms (also a marker of fibrosis) in the voltage range of even 1.5-2.5 mV. A similar approach has also been put forward by other groups. Yang et al. have examined 86 consecutive patients with non-paroxysmal AF (NPAF) in whom they performed high-density LA mapping in sinus rhythm, following ablation and cardioversion. They used similar definitions of low atrial voltage: 0.1 to $0.4 \mathrm{mV}$ were used to define low-voltage zone and bipolar voltage range of 0.4 to $1.3 \mathrm{mV}$ was chosen to represent transitional zones (34).

\section{Technical aspects of voltage mapping: role of underlying rhythm}

The underlying rhythm during mapping appears to be an important factor determining atrial voltage. In an early study Ndrepepa et al. demonstrate that bipolar signals during AF demonstrate significantly lower amplitudes in both atria as compared to sinus rhythm (35). Teh et al. studied 12 patients with persistent AF undergoing ablation. All the patients had high-density electroanatomical maps collected during AF and atrial pacing from the coronary sinus following AF conversion. The authors demonstrate that mean atrial voltages were significantly lower during $\mathrm{AF}(0.7 \pm 0.2 \mathrm{mV}$ vs. $2.1 \pm 0.6 \mathrm{mV}$, $P<0.001)$. Another series of 9 AF patients undergoing high-density LA mapping during AF, sinus rhythm and coronary sinus pacing has been reported by Jadidi et al. (36). Their data shows that mean LA voltage during is dependent on the type of arrhythmia with paroxys- mal AF patients showing significantly higher LA voltages $(1.12 \pm 1.32 \mathrm{mV})$ compared to persistent AF cases $(0.6 \pm 0.59 \mathrm{mV}), \mathrm{P}<0.01$. On the other hand, voltages at all mapping sites recorded during organised atrial rhythm (sinus or coronary sinus pacing) were $>1 \mathrm{mV}$ with mean LA voltage being significantly higher than the ones recorded during paroxysmal or persistent AF. Zones of low amplitudes in AF demonstrated normal EGM amplitude in sinus rhythm. In addition, there was no correlation between electrogram fractionation and LA voltage during organised atrial rhythm suggesting the purely functional nature of low voltage and EGM fractionation in AF patients. Similar results have been reported by Masuda et al. in a group of 30 patients with persistent AF (37). According to their data electrogram amplitudes during sinus rhythm were higher than those during AF $(1.77 \pm 1.27 \mathrm{mV}$ vs. $0.96 \pm 0.77$ $\mathrm{mV}, \mathrm{P}<0.0001)$. For each specific site electrogram amplitude during AF correlated only weakly with the amplitudes in sinus rhythm. The authors concluded that in patients with persistent AF there are significant disparities between electrogram amplitudes during sinus rhythm and AF at the same locations. Electrogram amplitudes recorded during organised atrial rhythm might also be variable depending on rate and direction of activation of the atrial myocardium. In a recent study, Wong et al. examine atrial substrate characteristics during pacing from the left superior pulmonary vein and from the coronary sinus at two different cycle lengths in $73 \mathrm{AF}$ patients (38). Mean atrial voltages were lower throughout the atria during coronary sinus pacing with $300 \mathrm{msec}$ as compared to $600 \mathrm{msec}(1.56 \pm 0.47 \mathrm{mV}$ vs. $1.74 \pm 0.48 \mathrm{mV}, \mathrm{P}<0.001)$. Furthermore, left superior pulmonary vein pacing at $300 \mathrm{msec}$ resulted in even lower mean LA voltage as compared to coronary sinus pacing at the same cycle length and introduced more expressed voltage heterogeneity in the LA. In contrast to that in a cohort of $27 \mathrm{AF}$ patients, Yagishita et al. reported a good correlation of electrogram amplitudes during AF and sinus rhythm. They also demonstrated lower mean LA bipolar voltage measured at identical or adjacent locations in persistent compared to paroxysmal AF measured both in AF $(0.81 \pm 0.60$ $\mathrm{mV}$ vs. $0.58 \pm 0.62 \mathrm{mV}, \mathrm{P}<0.001$ ) and in sinus rhythm $(2.24 \pm 1.51 \mathrm{mV}$ vs. $1.56 \pm 1.53 \mathrm{mV}, \mathrm{P}<0.001)$ (39). These findings support the notion that persistent AF is associated with more expressed atrial substrate which is not corroborated by other data as outlined above in the section on importance of AF phenotype. Another very recent study looks into the correlation of $L A$ voltage during sinus rhythm and $\mathrm{AF}$ with zones of atrial fibrosis assessed by DE-MRI in 20 patients with persistent AF (40). Atrial voltage assessed from multiple cycles during 8 second AF recordings was compared pairwise with the voltage at the same zones during sinus rhythm and correlated with the distribution of zones of delayed enhancement. The authors found a strong 
correlation between LA voltages during sinus rhythm and those recorded during AF. When correlated with the distribution of fibrotic areas values recorded during AF demonstrated better diagnostic accuracy to predict fibrosis. A voltage cut-off of $0.35 \mathrm{mV}$ in AF was found to detect fibrosis with a sensitivity and specificity of $75 \%$ and $79 \%$, respectively. In contrast, a threshold of $1.8 \mathrm{mV}$ during sinus rhythm was able to identify zones of delayed enhancement with a sensitivity of $63 \%$ and sensitivity of $67 \%$. A possible explanation for these results might be that low voltage during sinus rhythm identifies only zones where more coordinated wave fronts of activation encounter areas of conduction block and electrically inactivity typical for zones of compact fibrosis. The latter is only a part of the arrhythmogenic substrate identified by DE-MRI. During AF rapid and uncoordinated wave fronts spreading in areas of noncompact fibrosis which are susceptible to slow conduction may be associated with the occurrence of low voltage. Validation of a voltage cut-off with mapping during AF has also been attempted by a recent study including 31 AF patients where electrogram amplitudes of $0.31 \mathrm{mV}$ recorded during AF predicted minimal sinus rhythm electrogram amplitude of $0.5 \mathrm{mV}$ with a sensitivity of 0.82 and a specificity of 0.95 (41).

These numerous caveats and limitations are a powerful call for new studies to elucidate the voltage cut-offs used in LA voltage mapping and standardise the methodology by which LA voltage mapping is carried out. Further unknowns are introduced by many other factors which might have an impact on the results during LA voltage mapping. Among those electrode size and spacing, mapping density and tissue contact do play a considerable role.

\section{Studies of tailored ablation approach based on atrial voltage}

The approach to AF ablation targeting low voltage areas has been developing rapidly over the last 5 years. Among the first papers published on this topic is the one by Rolf et al. which provides interesting data from a non-randomised study (32). This study involved 178 AF patients all of which underwent detailed bipolar voltage map of the LA. All patients with low voltage $(<0.5 \mathrm{mV})$ underwent additional substrate modification in addition to PVI. It included regional ablation at the low voltage zones (LVZ) aiming at substrate homogenisation meant to achieve defractionation, significant reduction of local electrograms and loss of capture while pacing with high energy output. In many cases linear lesions traversing through the diseased areas and connecting nonconducting zones were also performed. Larger LVZs were encircled and electrically isolated. The study also included a control group of 26 patients with low voltage areas who underwent PVI only. In the study population LVZs were most frequently detected at the LA roof, anterior, posterior wall and septum and were more common in persistent cases. Ablation outcomes in patients with tailored substrate modification following PVI were comparable to those without LVZs undergoing PVI only. The control group had a poor outcome of a PVI strategy only. Results from this study generated hypotheses which were further addressed by numerous subsequent studies (Table 1) $(33,34,42-46)$. Most of these were observational and examined the outcomes of standard ablation in comparison to voltage guided ablation strategies in consecutive patients regardless of the presence of LVZs or compared outcomes of standard ablation versus standard ablation plus additional voltage-guided substrate modification in patients with LVZs.

Generally, a couple of ablation strategies have been used in all these studies (Figure 1). Two of the papers coming from the same group report the so-called box isolation of fibrotic areas (BIFA). With this technique the areas in both atria affected by fibrosis as detected by electrogram amplitudes $<0.5 \mathrm{mV}$ were encircled by circumferential lesions. They were usually connected to pulmonary venous isolation or other lesion sets to prevent occurrence of iatrogenic macroreentry circuits. The endpoint of BIFA was bidirectional block to and from the fibrotic area. This approach was tested in a small observational study of $41 \mathrm{AF}$ patients (33). Ten of them were paroxysmal AF patients undergoing redo procedures for arrhythmia recurrence despite durable PVI where BIFA was reported to result in arrhythmia freedom in $90 \%$ of the cases. The rest of the patients were ablation-naïve NPAF patients. Thirteen of them (42\%) underwent PVI only as they did not demonstrate LVZs in the LA (FACM 0 and 1). The remaining 18 patients demonstrated fibrotic areas (FACM 2 to 4 ) and underwent additional BIFA. After a follow-up of $12.5 \pm 2.4$ months multiple procedure efficacy in this patient population was $83.3 \%$. BIFA was indicated but impossible to perform due to very diffuse fibrosis in only one patient. These results give two very important messages. First, in about $40 \%$ of the NPAF patients in whom no LVZs are identified a strategy including PVI only appears to be effective. Second, there are patients with diffuse and dense fibrosis (FACM 4) in whom extensive ablation approaches of any kind should be discouraged because of low chance for long-term success. The same group extended their work to a larger series of patients. Schreiber et al. analysed the impact of LA fibrosis as assessed by electrogram voltage with regards to its distribution and rhythm outcome (42). This study included 49 patients without low voltage areas (FACM 0 ) who underwent PVI only and served as controls to 92 patients with FACM 1 to 4 who underwent additional BIFA. Overall long-term success rate from the study group was $84 \%$ after a single procedure and $94 \%$ following multiple procedures. The success rate was clearly dependent on FACM grade with FACM 3 and 4 patients demonstrating a consider- 


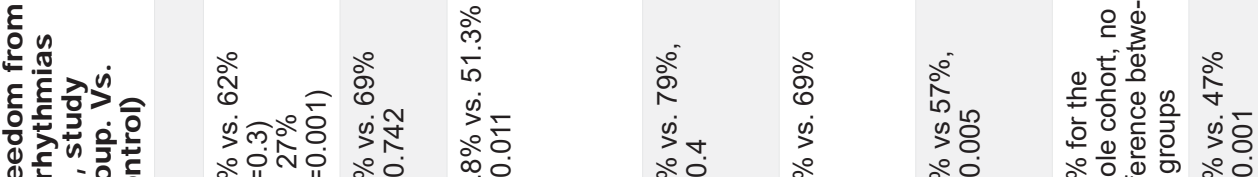

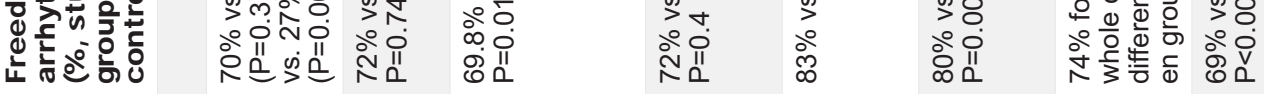

站点

$\simeq \quad \simeq \quad$

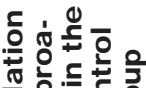

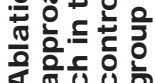

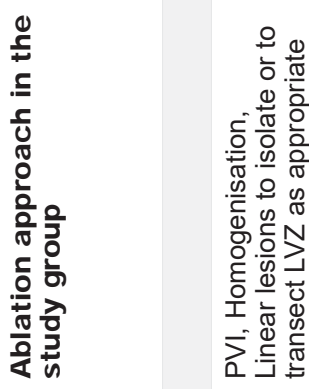

$>\quad \frac{\infty}{2}$

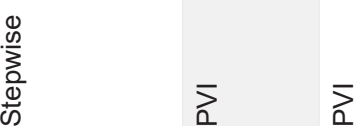

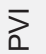

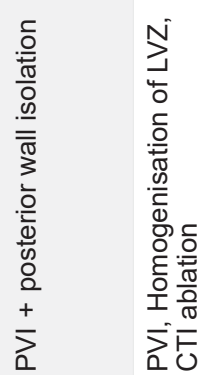

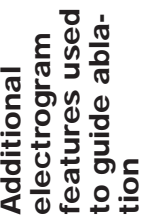

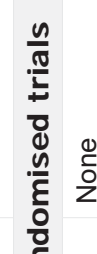

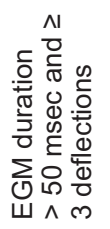

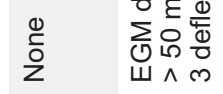

$\stackrel{0}{0} \frac{0}{2}$

$\stackrel{0}{\circ}$

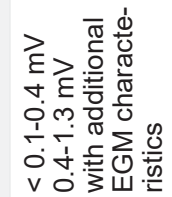

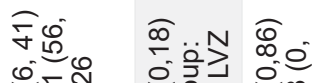

舟监

III

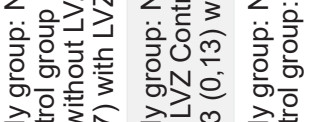

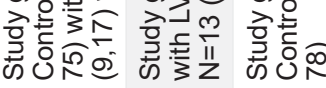

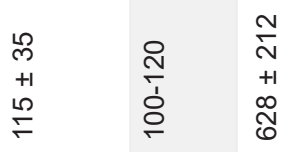

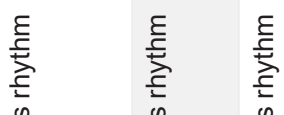

के के के

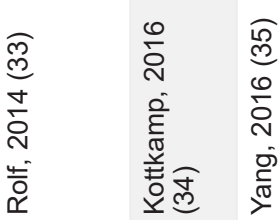

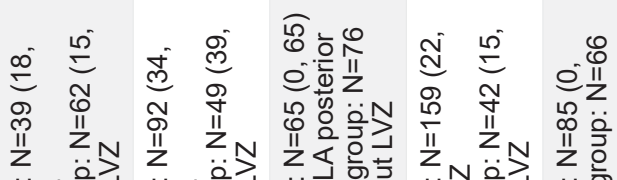

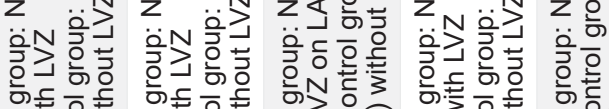

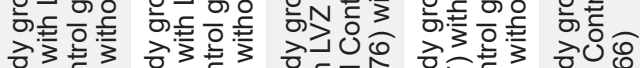

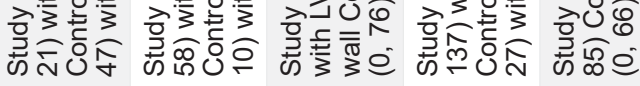

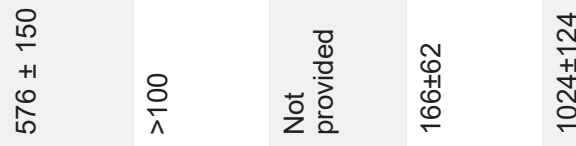

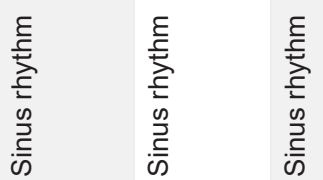

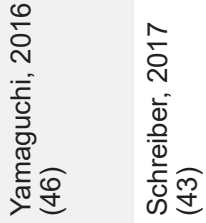

岁崖

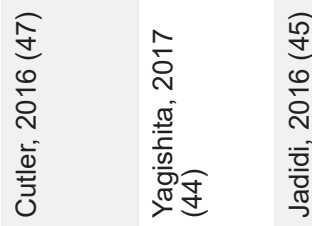

竞 $\stackrel{m}{-}$

হ

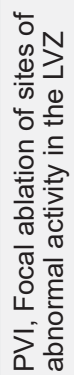

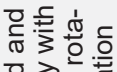

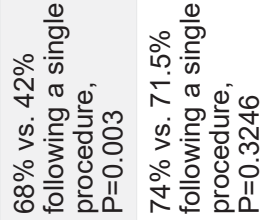

$\stackrel{m}{\stackrel{\infty}{\sim}} \stackrel{\infty}{\sim}$

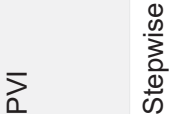

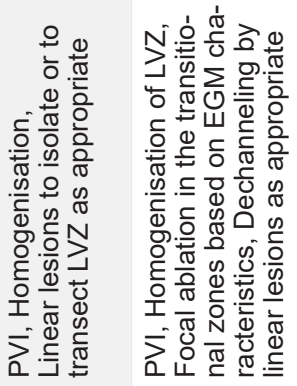

돈 닌

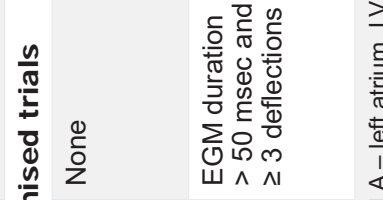

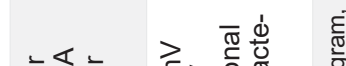

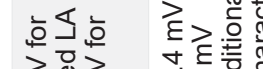

E

นึ.

o

这

인

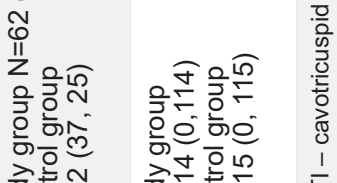

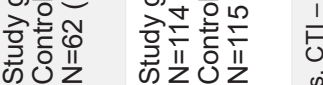

갓

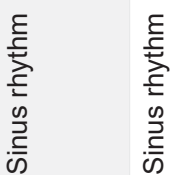

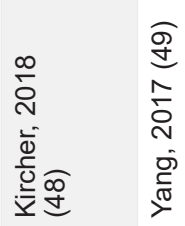



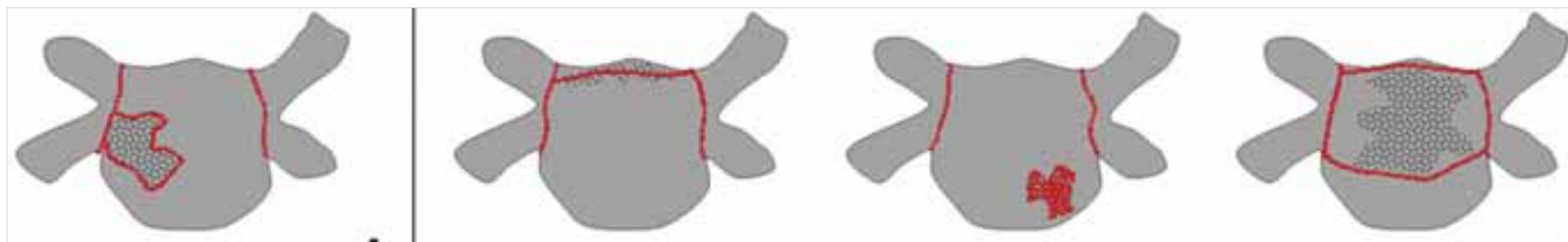

A
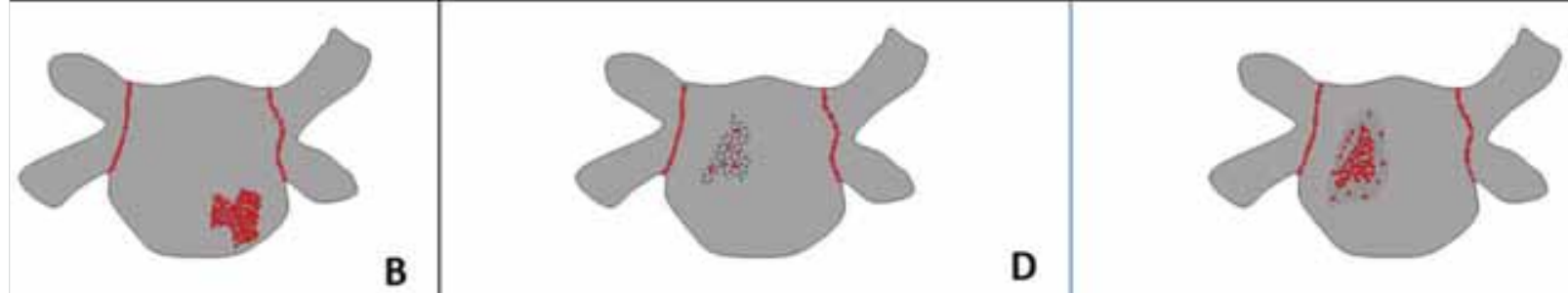

$\mathbf{E}$

Figure 1. Ablation strategies for individualised ablation based on LA voltage proposed by different groups. Panel A: Box isolation of fibrotic areas as described by Kottkamp et al. and Schreiber et al. (34, 43). Panel B: Homogenisation of low voltage areas as proposed by Yagishita et al. and Yamaguchi et al. $(44,46)$. Panel C: Combined approach using linear ablation transecting diseased areas (left panel), regional ablation targeting homogenisation of small low voltage areas (middle panel) and encirclement of larger low voltage areas by linear lesions connected to other non-conducting barriers e.g. PVI lesion sets or mitral annulus (right panel) as proposed by Rolf et al. and Kircher et al. $(33,47)$ Panel D Combined approach using identification of low voltage zones and focal ablation to sites of abnormal electrical activity as suggested by Jadidi et al. (45), see text for details. Panel E. Combined approach targeting homogenisation of low-voltage zones (0.1-0.4 mV) and elimination of abnormal electrograms in the transitional zone $(0.4-1.3 \mathrm{mV})$ as used by Yang et al. $(35,48)$, see text for further details

ably lower success rates as compared to FACM 1 and 2. Not surprisingly, the lower success rate in the former group was driven by the FACM 4 patients who demonstrated a success rate of only $20 \%$.

Yagishita et al. analysed $201 \mathrm{AF}$ patients (persistent in $82 \%$ ) who underwent electroanatomical mapping of the LA during AF (43). With a voltage cut-off of $<0.5 \mathrm{mV}$ LVZs were identified in $79 \%$ of the patients who underwent additional substrate modification - homogenisation and cavotricuspid isthmus ablation as appropriate. At 12 months and following a single procedure the arrhythmia free rate for the whole study population was $72 \%$. After a long follow-up of 3.1 years success rate for the whole cohort after multiple procedures was $74 \%$. There was no difference in the success rate between patients without LVZs undergoing $\mathrm{PVI}$ only and those with LVZs who underwent PVI and substrate modification. One of the major and debatable findings this study is the high prevalence of LVZs (79\%) which might be a result of mapping during AF. Jadidi et al. attempted to combine low-voltage and activation criteria during AF (44). They studied 85 NPAF patients who underwent voltage mapping during $A F$ and subsequent $P V I$. When low voltage zones $(<0.5 \mathrm{mV})$ were identified in the LA or right atrium, additional focal ablation was applied to those sites where electrical activity lasting more than $70 \%$ of AF cycle length on single or multiple electrodes from a confined area. This strategy was applied to 62 patients from the study cohort, $73 \%$ of whom had AF termination to sinus rhythm or atrial tachycardia. Following a median of 13 months of follow up $69 \%$ of the patients treated with $\mathrm{PVI}$ and additional selective atrial ablation were free from arrhythmia compared to only $47 \%$ in the patients with PVI-only approach ( $P<0.001)$. Interestingly, the sites where AF terminated were found in LVZs in $80 \%$ of the cases and $20 \%$ were located at border zones.

Important findings have been reported by Yang et al. who studied 164 patients with NPAF (34). Eighty-six of those were treated with PVI and cavotricuspid isthmus ablation and subsequent substrate modification strategy including ablation of all electrograms in LVZ (0.1-0.4 $\mathrm{mV}$ ) aiming to achieve substrate homogenisation. In addition, abnormal electrograms consisting of multiphasic electrograms with $>3$ distinct peaks and $>50$ msec duration in the so-called transitional zone (0.4-1.3 mV) were also targeted and eliminated. Short linear lesions were also placed to achieve dechanelling. A control group of patients underwent a stepwise ablation approach consisting of $\mathrm{PVI}$, followed in a stepwise manner by linear lesions and complex-fractionated electrogram ablation until AF termination. Long term success rates were significantly higher in the study group: $70 \%$ vs. $51 \%$, respectively, $P=0.011$. Another important result reported by these investigators was the significantly higher rate of postprocedural atrial tachycardia in the control group (30\% vs. $3.5 \%$ in the study group, $P=0.0003$ ). Similar findings were reported by Yamaguchi et al. in a series of patients with NPAF undergoing PVI and homogenisation of LVZs (45). Thirty-nine of the studied patients had LA LVZs ( $<0.5 \mathrm{mV})$. They underwent PVI and subsequent substrate modification. The remaining 62 patients had no LVZs and underwent PVI only. In the two groups single procedure long-term success rates were 
similar $-72 \%$ and $79 \%$ respectively. In contrast, in an additional group of patients with LVZs who underwent PVI only the long-term success rate was only $38 \%$.

Direct comparison among these studies is difficult to make as they report quite heterogenous data in terms of mapping/ablation strategies, prevalence of LVZs, patient profile etc. In attempt to overcome these limitations Blandino et al. have combined data from six studies in a meta-analysis. Altogether 885 patients were enrolled of whom $92 \%$ had NPAF. Results from the combined strategy of PVI and voltage-guided ablation were superior when compared to the standard approach (PVI or $\mathrm{PVI}+$ empirical substrate ablation) in terms of long-term arrhythmia freedom $-70 \%$ vs. $43 \%$ respectively (OR = $3.41,95 \% \mathrm{Cl} 2.22-5.24)$ without a significant impact on adverse events rate. This strategy was also associated with lower occurrence of postablation atrial tachycardias, shorter procedure duration and fluoroscopy times. Two randomised studies with the voltage-guided ablation strategy have also been performed with inconsistent and contrasting results. Kircher et al. randomised 124 AF patients to PVI or PVI and additional linear ablation (for persistent cases only) or to PVI combined with ablation of LVZs (<0.5 mV in sinus rhythm) (47). The ablation strategy that was used was identical to the one proposed by Rolf et al. (32). Following a mean follow-up of $12 \pm 3$ months significantly more patients in the voltage guided ablation group met the primary endpoint of freedom from atrial arrhythmias off antiarrhythmic drugs after a single procedure (68\% vs. $42 \%, P=0.003$ ). Secondary efficacy endpoints (single procedure arrhythmia free survival on or off antiarrhythmic drugs and single procedure freedom from arrhythmias during 7-day Holter recording at 6 and 12 months) were also more common in the intervention group. Safety outcomes were comparable in the two groups. Contrary to that, Yang et al. reported similar long-term outcomes following a single procedure in the randomised STABLE SR trial which enrolled 229 symptomatic NPAF patients (48). They were randomised to PVI combined with tailored substrate modification versus stepwise approach. The former included homogenisation of low voltage zones (0.1-0.4 mV identified during sinus rhythm), elimination of complex electrograms from the transitional zones (0.4-1.3 mV during sinus rhythm) and dechanelling (34). The latter included PVI, followed in a stepwise fashion by linear ablation at the LA roof, the mitral isthmus and cavotricusid isthmus and subsequent ablation of complex fractionated electrograms in AF aiming at AF termination. In the intention-to-treat analysis the primary endpoint of freedom from AF or other atrial tachyarrhythmias at 18 months following a single procedure was not significantly different between the two groups (74\% for the tailored intervention group vs $71.5 \%$ for the stepwise approach, $\mathrm{P}=0.3246)$. However, the tailored ablation approach was associated with significantly shorter procedure and fluoroscopic times as well as reduced energy delivery time. A potential explanation for the lack of consistency between these two studies might be slight differences in the patient characteristics: the patients from the STABLE SR cohort were mainly persistent cases but likely with less diseased atria (low average age, non-dilated atria and low CHADS2 in the majority of the subjects). On the other hand, in the study by Kircher et al. the freedom from arrhythmia recurrence in the paroxysmal patients from the control group was lower than expected and previously reported which might have affected the results and strengthen the difference between the two groups.

\section{Conclusion}

Atrial fibrosis plays an important role in the complex pathophysiology of both paroxysmal and non-paroxysmal AF. Location and extent of fibrotic areas are highly variable and ablation strategies implementing empirical linear lesions or fragmented electrogram ablation might leave great parts of these areas untreated thus decreasing long-term ablation success. This holds true especially for the NPAF patients in which arrhythmogenic atrial substrate plays a greater role. On the other hand, some patients with less expressed substrate are overtreated with the potential risk of increased complication rates. Therefore, ablation strategies tailored to the substrate in each individual patient would provide one of the most effective rhythm control options in paroxysmal and non-paroxysmal AF. Voltage mapping is a rapid procedure providing intuitive data on the distribution of fibrotic areas in the atria by demonstration of LVZs. However, this technique is not devoid of limitations and some areas such as exact voltage cut-off values that could be utilised to identify fibrotic areas most accurately require further study. Despite this many observational studies demonstrate clear benefit from a voltage guided ablation approach. Randomised data are as yet inconclusive in terms of success but appears to show better procedural characteristics compared to the empirical approach. Further evidence from large prospective multicentre randomised trials is needed to establish the safety profile of such an approach and to further verify its efficacy as compared to standard ablation.

\section{Declaration of interest}

The author has reported that he has no relationships relevant to the contents of this paper to disclose.

\section{References}

1. Wijffels MC, Kirchhof CJ, Dorland R, Allessie MA. Atrial fibrillation begets atrial fibrillation. A study in awake chronically instrumented goats. Circulation 1995; 92(7): 1954-68. DOI: 10.1161/01. cir.92.7.1954 
2. Mahnkopf C, Badger TJ, Burgon NS, Daccarett M, Haslam TS, Badger CT, et al. Evaluation of the left atrial substrate in patients with lone atrial fibrillation using delayed-enhanced MRI implications for disease progression and response to catheter ablation. Heart Rhythm 2010; 7(10): 1475-81. DOI: 10.1016/j.hrthm.2010.06.030 3. Kirchhof P, Benussi S, Kotecha D, Ahlsson A, Atar D, Casadei B, et al. 2016 ESC Guidelines for the management of atrial fibrillation developed in collaboration with EACTS. Europace 2016; 18(11): 1609-78. DOI: 10.1093/ejcts/ezw313

4. Calkins H, Hindricks G, Cappato R, Kim YH, Saad EB, Aguinaga L, et al. 2017 HRS/EHRA/ECAS/APHRS/SOLAECE expert consensus statement on catheter and surgical ablation of atrial fibrillation. Europace 2018; 20(1): e1-e160. DOI: 10.1093/europace/eux274

5. Haissaguerre M, Jais P, Shah DC, Takahashi A, Hocini M, Quiniou $\mathrm{G}$, et al. Spontaneous initiation of atrial fibrillation by ectopic beats originating in the pulmonary veins. N Engl J Med 1998; 339(10): 659-66. DOI: 10.1056/NEJM199809033391003

6. Phlips T, Taghji P, El Haddad M, Wolf M, Knecht S, Vandekerckhove $Y$, et al. Improving procedural and one-year outcome after contact force-guided pulmonary vein isolation: the role of interlesion distance, ablation index, and contact force variability in the 'CLOSE'protocol. Europace 2018; 20(Fi_3): f419-f27.

7. Verma A, Jiang CY, Betts TR, Chen J, Deisenhofer I, Mantovan $\mathrm{R}$, et al. Approaches to catheter ablation for persistent atrial fibrillation. N Engl J Med 2015; 372(19): 1812-22. DOI: 10.1056/NEJMoa1408288

8. Nademanee K, McKenzie J, Kosar E, Schwab M, Sunsaneewitayakul B, Vasavakul T, et al. A new approach for catheter ablation of atrial fibrillation: mapping of the electrophysiologic substrate. J Am Coll Cardiol 2004; 43(11) 2044-53. DOI: 10.1016/j.jacc.2003.12.054 9. Pambrun T, Denis A, Duchateau J, Sacher F, Hocini M, Jais P, et al. MARSHALL bundles elimination, Pulmonary veins isolation and Lines completion for ANatomical ablation of persistent atrial fibrillation MARSHALL-PLAN case series. J Cardiovasc Electrophysiol 2019; 30(1): 7-15. DOI: 10.1111/jce.13797

10. Scherr D, Khairy P, Miyazaki S, Aurillac-Lavignolle V, Pascale P, Wilton SB, et al. Five-year outcome of catheter ablation of persistent atrial fibrillation using termination of atrial fibrillation as a procedural endpoint. Circ Arrhythm Electrophysiol. 2015; 8(1): 18-24. DOI: 10.1161/CIRCEP.114.001943

11. Narayan SM, Krummen DE, Shivkumar K, Clopton P, Rappel WJ, Miller JM. Treatment of atrial fibrillation by the ablation of localized sources: CONFIRM (Conventional Ablation for Atrial Fibrillation With or Without Focal Impulse and Rotor Modulation) trial. J Am Coll Cardiol. 2012; 60(7): 628-36. DOI: 10.1016/j.jacc.2012.05.022

12. Goette A, Kalman JM, Aguinaga L, Akar J, Cabrera JA, Chen SA, et al. EHRA/HRS/APHRS/SOLAECE expert consensus on Atrial cardiomyopathies: Definition, characterisation, and clinical implication. J Arrhythm 2016; 32(4): 247-78. DOI: 10.1016/j. joa.2016.05.002

13. Kottkamp H. Fibrotic atrial cardiomyopathy a specific disease/ syndrome supplying substrates for atrial fibrillation, atrial tachycardia, sinus node disease, AV node disease, and thromboembolic complications. J Cardiovasc Electrophysiol. 2012; 23(7): 797-9. DOI: 10.1111/j.1540-8167.2012.02341.x

14. Konrad T, Theis $C$, Mollnau $H$, Sonnenschein S, Ocete Bq, Bock K, et al. Primary Persistent Atrial Fibrillation: A Distinct Arrhythmia Subentity of an Ablation Population. Journal of Cardiovascular Electrophysiology 2015; 26(12): 1289-94. DOI: 10.1111/jce.12818

15. Lim HS, Denis A, Middeldorp ME, Lau DH, Mahajan R, Derval N, et al. Persistent Atrial Fibrillation From the Onset: A Specific Subgroup of Patients With Biatrial Substrate Involvement and Poor- er Clinical Outcome. JACC Clin Electrophysiol 2016; 2(2): 129-39. DOI: 10.1016/j.jacep.2015.12.014

16. Boldt A, Wetzel U, Lauschke J, Weigl J, Gummert J, Hindricks $\mathrm{G}$, et al. Fibrosis in left atrial tissue of patients with atrial fibrillation with and without underlying mitral valve disease. Heart 2004; 90(4): 400-5. DOI: 10.1136/hrt.2003.015347

17. Stiles MK, John B, Wong CX, Kuklik P, Brooks AG, Lau DH, et al. Paroxysmal lone atrial fibrillation is associated with an abnormal atrial substrate: characterizing the "second factor". J Am Coll Cardiol. 2009; 53(14): 1182-91. DOI: 10.1016/j.jacc.2008.11.054

18. McGann C, Akoum N, Patel A, Kholmovski E, Revelo P, Damal $\mathrm{K}$, et al. Atrial fibrillation ablation outcome is predicted by left atrial remodeling on MRI. Circ Arrhythm Electrophysiol 2014; 7(1): 23-30. DOI: 10.1161/CIRCEP.113.000689

19. Marrouche NF, Wilber D, Hindricks G, Jais P, Akoum N, Marchlinski $F$, et al. Association of atrial tissue fibrosis identified by delayed enhancement MRI and atrial fibrillation catheter ablation: the DECAAF study. Jama 2014; 311(5): 498-506. DOI: 10.1001/ jama.2014.3

20. Spragg DD, Khurram I, Zimmerman SL, Yarmohammadi $\mathrm{H}$, Barcelon B, Needleman M, et al. Initial experience with magnetic resonance imaging of atrial scar and co-registration with electroanatomic voltage mapping during atrial fibrillation: success and limitations. Heart Rhythm 2012; 9(12): 2003-9. DOI: 10.1016/j. hrthm.2012.08.039

21. Malcolme-Lawes LC, Juli C, Karim R, Bai W, Quest R, Lim PB, et al. Automated analysis of atrial late gadolinium enhancement imaging that correlates with endocardial voltage and clinical outcomes: a 2-center study. Heart Rhythm 2013; 10(8): 1184-91. DOI: 10.1016/j. hrthm.2013.04.030

22. Khurram IM, Beinart R, Zipunnikov V, Dewire J, Yarmohammadi $\mathrm{H}$, Sasaki $\mathrm{T}$, et al. Magnetic resonance image intensity ratio, a normalized measure to enable interpatient comparability of left atrial fibrosis. Heart Rhythm 2014; 11(1): 85-92. DOI: 10.1016/j. hrthm.2013.10.007

23. Park JH, Pak HN, Choi EJ, Jang JK, Kim SK, Choi DH, et al. The relationship between endocardial voltage and regional volume in electroanatomical remodeled left atria in patients with atrial fibrillation: comparison of three-dimensional computed tomographic images and voltage mapping. J Cardiovasc Electrophysiol 2009; 20(12): 1349-56. DOI: 10.1111/j.1540-8167.2009.01557.x

24. Park J, Joung B, Uhm JS, Young Shim C, Hwang C, Hyoung Lee $M$, et al. High left atrial pressures are associated with advanced electroanatomical remodeling of left atrium and independent predictors for clinical recurrence of atrial fibrillation after catheter ablation. Heart Rhythm 2014; 11(6): 953-60. DOI: 10.1016/j. hrthm.2014.03.009

25. Sung SH, Chang SL, Hsu TL, Yu WC, Tai CT, Lin YJ, et al. Do the left atrial substrate properties correlate with the left atrial mechanical function? A novel insight from the electromechanical study in patients with atrial fibrillation. J Cardiovasc Electrophysiol 2008; 19(2): 165-71. DOI: 10.1111/j.1540-8167.2007.00982.x

26. Watanabe $Y$, Nakano $Y$, Hidaka T, Oda N, Kajihara K, Tokuyama $\mathrm{T}$, et al. Mechanical and substrate abnormalities of the left atrium assessed by 3-dimensional speckle-tracking echocardiography and electroanatomic mapping system in patients with paroxysmal atrial fibrillation. Heart Rhythm 2015; 12(3): 490-7. DOI: 10.1016/j. hrthm.2014.12.007

27. Yagishita A, Sparano D, Cakulev I, Gimbel JR, Phelan T, Mustafa $\mathrm{H}$, et al. Identification and electrophysiological characterization of early left atrial structural remodeling as a predictor for atrial fibrillation recurrence after pulmonary vein isolation. J Cardiovasc Electro- 
physiol 2017; 28(6): 642-50. DOI: 10.1111/jce.13211

28. Saghy L, Callans DJ, Garcia F, Lin D, Marchlinski FE, Riley M, et al. Is there a relationship between complex fractionated atrial electrograms recorded during atrial fibrillation and sinus rhythm fractionation? Heart Rhythm 2012; 9(2): 181-8. DOI: 10.1016/j. hrthm.2011.09.062

29. Teh AW, Kistler PM, Lee G, Medi C, Heck PM, Spence SJ, et al. Electroanatomic remodeling of the left atrium in paroxysmal and persistent atrial fibrillation patients without structural heart disease. $J$ Cardiovasc Electrophysiol 2012; 23(3): 232-8. DOI: 10.1111/j.15408167.2011.02178.x

30. Verma A, Wazni OM, Marrouche NF, Martin DO, Kilicaslan F, Minor S, et al. Pre-existent left atrial scarring in patients undergoing pulmonary vein antrum isolation: an independent predictor of procedural failure. J Am Coll Cardiol 2005; 45(2): 285-92. DOI: 10.1016/j. jacc.2004.10.035

31. Kapa S, Desjardins B, Callans DJ, Marchlinski FE, Dixit S. Contact electroanatomic mapping derived voltage criteria for characterizing left atrial scar in patients undergoing ablation for atrial fibrillation. J Cardiovasc Electrophysiol 2014; 25(10): 1044-52. DOI: 10.1111/jce.12452

32. Rolf S, Kircher S, Arya A, Eitel C, Sommer P, Richter S, et al. Tailored atrial substrate modification based on low-voltage areas in catheter ablation of atrial fibrillation. Circ Arrhythm Electrophysiol 2014; 7(5): 825-33. OI: 10.1161/CIRCEP.113.001251

33. Kottkamp H, Berg J, Bender R, Rieger A, Schreiber D. Box Isolation of Fibrotic Areas (BIFA): A Patient-Tailored Substrate Modification Approach for Ablation of Atrial Fibrillation. J Cardiovasc Electrophysiol. 2016; 27(1): 22-30. DOI: 10.1111/jce.12870

34. Yang G, Yang B, Wei Y, Zhang F, Ju W, Chen H, et al. Catheter Ablation of Nonparoxysmal Atrial Fibrillation Using Electrophysiologically Guided Substrate Modification During Sinus Rhythm After Pulmonary Vein Isolation. Circ Arrhythm Electrophysiol 2016; 9(2): e003382. DOI: 10.1161/CIRCEP.115.003382

35. Ndrepepa G, Schneider MA, Karch MR, Weber S, Schreieck J, Zrenner B, et al. Impact of atrial fibrillation on the voltage of bipolar signals acquired from the left and right atria. Pacing Clin Electrophysiol 2003; 26(4 Pt 1): 862-9. DOI: 10.1046/j.1460-9592.2003. t01-1-00151.x

36. Jadidi AS, Duncan E, Miyazaki S, Lellouche N, Shah AJ, Forclaz $A$, et al. Functional nature of electrogram fractionation demonstrated by left atrial high-density mapping. Circ Arrhythm Electrophysiol 2012; 5(1): 32-42. DOI: 10.1161/CIRCEP.111.964197

37. Masuda M, Fujita M, lida O, Okamoto $S$, Ishihara $T$, Nanto $K$, et al. Comparison of Left Atrial Voltage between Sinus Rhythm and Atrial Fibrillation in Association with Electrogram Waveform. Pacing Clin Electrophysiol 2017; 40(5): 559-67. DOI: 10.1111/pace.13051

38. Wong GR, Nalliah CJ, Lee G, Voskoboinik A, Prabhu S, Parameswaran R, et al. Dynamic Atrial Substrate During High-Density Mapping of Paroxysmal and Persistent AF: Implications for Substrate Ablation. JACC Clin Electrophysiol 2019; 5(11): 1265-77. DOI: 10.1016/j.jacep.2019.06.002

39. Yagishita A, S DEO, Cakulev I, Gimbel JR, Sparano D, Manyam $\mathrm{H}$, et al. Correlation of Left Atrial Voltage Distribution Between Sinus Rhythm and Atrial Fibrillation: Identifying Structural Remodeling by 3-D Electroanatomic Mapping Irrespective of the Rhythm. J Cardiovasc Electrophysiol 2016; 27(8): 905-12. DOI: 10.1111/ jce.13002

40. Qureshi NA, Kim SJ, Cantwell CD, Afonso VX, Bai W, Ali RL, et al. Voltage during atrial fibrillation is superior to voltage during sinus rhythm in localizing areas of delayed enhancement on magnetic resonance imaging: An assessment of the posterior left atrium in patients with persistent atrial fibrillation. Heart Rhythm 2019; 16(9): 1357-67. DOI: 10.1016/j.hrthm.2019.05.032

41. Rodriguez-Manero $M$, Valderrabano $M$, Baluja $A$, Kreidieh $O$, Martinez-Sande JL, Garcia-Seara J, et al. Validating Left Atrial Low Voltage Areas During Atrial Fibrillation and Atrial Flutter Using Multielectrode Automated Electroanatomic Mapping. JACC Clin Electrophysiol 2018; 4(12): 1541-52. DOI: 10.1016/j.jacep.2018.08.015

42. Schreiber D, Rieger A, Moser F, Kottkamp H. Catheter ablation of atrial fibrillation with box isolation of fibrotic areas: Lessons on fibrosis distribution and extent, clinical characteristics, and their impact on long-term outcome. J Cardiovasc Electrophysiol 2017; 28(9): 971-83. DOI: 10.1111/jce.13278

43. Yagishita A, Gimbel JR, S DEO, Manyam H, Sparano D, Cakulev I, et al. Long-Term Outcome of Left Atrial Voltage-Guided Substrate Ablation During Atrial Fibrillation: A Novel Adjunctive Ablation Strategy. J Cardiovasc Electrophysiol 2017; 28(2): 147-55. DOI: 10.1111/ jce.13122

44. Jadidi AS, Lehrmann H, Keyl C, Sorrel J, Markstein V, Minners J, et al. Ablation of Persistent Atrial Fibrillation Targeting Low-Voltage Areas With Selective Activation Characteristics. Circ Arrhythm Electrophysiol 2016; 9(3). DOI: 10.1161/CIRCEP.115.002962

45. Yamaguchi $T$, Tsuchiya $T$, Nakahara $S$, Fukui $A$, Nagamoto $Y$, Murotani K, et al. Efficacy of Left Atrial Voltage-Based Catheter Ablation of Persistent Atrial Fibrillation. J Cardiovasc Electrophysiol 2016; 27(9): 1055-63. DOI: 10.1111/jce.13019

46. Cutler MJ, Johnson J, Abozguia K, Rowan S, Lewis W, Costantini O, et al. Impact of Voltage Mapping to Guide Whether to Perform Ablation of the Posterior Wall in Patients With Persistent Atrial Fibrillation. J Cardiovasc Electrophysiol 2016; 27(1): 13-21. DOI: 10.1111/ jce.12830

47. Kircher S, Arya A, Altmann D, Rolf S, Bollmann A, Sommer P, et al. Individually tailored vs. standardized substrate modification during radiofrequency catheter ablation for atrial fibrillation: a randomized study. Europace 2018; 20(11): 1766-75. DOI: 10.1093/europace/eux310

48. Yang B, Jiang $C$, Lin $Y$, Yang $G$, Chu $H$, Cai H, et al. STABLE-SR (Electrophysiological Substrate Ablation in the Left Atrium During Sinus Rhythm) for the Treatment of Nonparoxysmal Atrial Fibrillation: A Prospective, Multicenter Randomized Clinical Trial. Circ Arrhythm Electrophysiol 2017; 10(11): DOI: 10.1161/CIRCEP.117.005405 